\title{
Pulmonary Thromboembolism and Diffuse Alveolar Hemorrhage in Granulomatosis With Polyangiitis Vasculitis
}

\author{
Gabriel Moreno-González MD PhD, Luisa Corral-Ansa MD PhD, Joan Sabater-Riera MD, \\ Xavier Solanich-Moreno MD, and Rafael Mañez-Mendiluce MD PhD
}

\section{Introduction}

Granulomatosis with polyangiitis (GPA) is a small-vessel and medium-vessel vasculitis that affects mainly the respiratory tract and kidney. The main acute complications of GPA are diffuse pulmonary hemorrhage and acute renal failure. Renal involvement occurs in $\sim 17 \%$ of patients, with histological features of pauci-immune, focal, and segmental necrotizing crescentic glomerulonephritis. ${ }^{1}$ Diffuse alveolar hemorrhage (DAH) is the most severe complication in small-vessel and medium-vessel vasculitis, and immunosuppressive therapy alone is not sufficient to treat this condition. A recent study revealed that the incidence of DAH in anti-neutrophil cytoplasmic antibody-associated vasculitis (AAV) ranges from 8 to $36 \%$, with $41 \%$ of these diagnosed as GPA. ${ }^{2}$ There is no consensus for the diagnostic criteria of DAH, but the presence of dyspnea, hemoptysis, $\mathrm{P}_{\mathrm{aO}} / \mathrm{F}_{\mathrm{IO}_{2}}<300$, a decrease in hemoglobin levels (between 1 and $2 \mathrm{~g} / \mathrm{dL}$ ), compatible bronchoscopy or bronchoalveolar lavage, and abnormal chest radiography or computed tomography have been reported in different studies. ${ }^{2}$ Respiratory failure due to DAH and sepsis are the most common reasons for ICU admission in these patients, with $28-\mathrm{d}$ and $1-\mathrm{y}$ mortality rates of $11 \%$ and $29 \%$, respectively, in a retrospective cohort study including 26 subjects. ${ }^{3}$ Moreover, there is an increased incidence of thromboembolic disease in GPA vasculitis. ${ }^{4,5}$ Here, we report the case of a patient with active GPA, anticoagu-

Drs Moreno-González, Corral-Ansa, Sabater-Riera, and Mañez-Mendiluce are affiliated with the Critical Care Unit, and Dr Solanich-Moreno is affiliated with the Internal Medicine Department, Hospital Universitari de Bellvitge, Barcelona, Spain.

The authors have disclosed no conflicts of interest.

Correspondence: Gabriel Moreno-González MD PhD, Critical Care Unit, Hospital Universitari de Bellvitge, Feixa Llarga S/N, 08907 Hospitalet de Llobregat, Barcelona, Spain. E-mail: gabriel.moreno@bellvitge hospital.cat.

DOI: $10.4187 /$ respcare. 03162 lated because of deep venous thrombosis and pulmonary emboli, who developed DAH and renal failure.

\section{Case Summary}

A 42-y-old man was admitted to the ICU with dyspnea, hemoptysis, and renal failure. He was diagnosed with GPA $2 \mathrm{y}$ before admission and treated with prednisone $(30 \mathrm{mg} / \mathrm{d})$ and azathioprine $(50 \mathrm{mg}, 3$ times/d), developing diabetes secondary to corticoid use. He had 3 episodes of intestinal vasculitis. The initial 2 episodes were medically treated, whereas the latter required laparoscopic sigmoidectomy the year prior to admission. He was diagnosed with deep venous thrombosis 2 y before admission and initially treated with low-weight heparin and then with oral anticoagulants thereafter. Five months before admission, he voluntarily stopped oral anticoagulation and his regular medication. Because of an increase in right leg volume and dyspnea on exertion he was diagnosed with deep venous thrombosis with pulmonary thromboembolism 1 month before admission. He was initially treated with low-weight heparin and then with oral anticoagulants.

He presented to the emergency department of our hospital because of a 3-d history of minimal effort dyspnea and hemoptysis. On admission, the patient had severe hypoxemic respiratory insufficiency with a $\mathrm{P}_{\mathrm{aO}_{2}} / \mathrm{F}_{\mathrm{IO}_{2}}$ of 53 and impaired renal function with serum creatinine of 3.72 $\mathrm{mg} / \mathrm{dL}(0-1.19)$, urea of $58.5 \mathrm{mg} / \mathrm{dL}(10-24)$, and hyperglycemia of $354.9 \mathrm{mg} / \mathrm{dL}$ (73-124). The complete blood count revealed acute anemia with hemoglobin of $9.2 \mathrm{~g} / \mathrm{dL}$, a hematocrit of $27 \%$, and normal platelet and leukocyte counts. A coagulation test revealed an international normalized ratio of $2.8(0.8-1.2)$ (subject was on acenocoumarol) and a partial thromboplastin time of $0.85(0.8-1.2)$. Titers were as follows: anti-nuclear antibody, 1:40; anticardiolipin antibody, 2 GPL units (0-12); anti-myeloperoxidase antibody, 0.3 arbitrary concentration unit/L (0-7); and anti-proteinase 3 antibody, $>100$ arbitrary concentration units/L (0-7). Urinary analysis showed albuminuria of $107 \mathrm{~g} / \mathrm{mol}(0-3.4)$. Chest radiography showed bilateral alveolar infiltrates (Fig. 1A), and computed tomography 


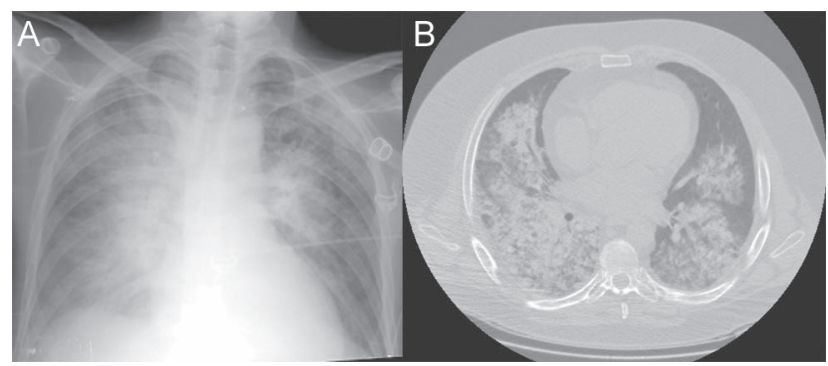

Fig. 1. A: Chest radiography showing bilateral alveolar opacities on ICU admission. B: Computed tomography showing bilateral areas of consolidation interspersed with areas of ground-glass attenuation and preserved normal areas.

revealed an alveolar occupation of the right lung and superior lobe of the left lung suggestive of alveolar hemorrhage (Fig. 1B).

Once the patient was in the ICU, noninvasive ventilation (NIV) was started with an $\mathrm{F}_{\mathrm{IO}_{2}}$ of 1 , PEEP of $6 \mathrm{~cm} \mathrm{H}_{2} \mathrm{O}$, and support pressure of $10 \mathrm{~cm} \mathrm{H}_{2} \mathrm{O}$. Cardiac echography did not reveal right ventricular failure, and ultrasonography of lower limbs showed right femoral deep venous and gastrocnemius vein thrombosis. Anticoagulation was stopped, and intra-arterial digital subtraction angiography was performed to place a filter in the inferior vena cava. High doses of intravenous corticosteroids, oral cyclophosphamide, and plasmapheresis were initiated the same day of admission. The complete treatment included $1 \mathrm{~g}$ of intravenous methylprednisolone for $3 \mathrm{~d}$ and then $1 \mathrm{mg} / \mathrm{kg} / \mathrm{d}$, oral cyclophosphamide at $2 \mathrm{mg} / \mathrm{kg} / \mathrm{d}$, and daily plasmapheresis for 1 week and then 3 times per week for another week. Trimethoprim/sulfamethoxazole at 800/160 mg was also given 3 times per week for Pneumocystis jiroveci prophylaxis. The patient required transfusion of one pack of red blood cells due to hemoglobin levels $(6.5 \mathrm{mg} / \mathrm{dL})$. He stayed in the ICU for $9 \mathrm{~d}$; his respiratory function improved, and he was weaned from NIV. After 3 weeks in the internal medicine department, the patient was discharged from the hospital, with improved respiratory and renal function (serum creatinine of $2.14 \mathrm{mg} / \mathrm{dL}$ ).

\section{Discussion}

According to the European Vasculitis Study, active GPA disease is classified as localized, early systemic, generalized, severe, or refractory depending on organ involvement and severity of disease. ${ }^{6} \mathrm{We}$ classified our patient as severe active GPA because of 2-organ involvement (severe respiratory failure with $\mathrm{P}_{\mathrm{aO}_{2}} / \mathrm{F}_{\mathrm{IO}_{2}}<100$ and serum creatinine levels of $>1.39 \mathrm{mg} / \mathrm{dL}$ ). Guidelines recommend that after diagnosis, classification, and initial assessment, early initiation of remission-induction therapy is impera- tive for these patients. A combination of oral cyclophosphamide $(2 \mathrm{mg} / \mathrm{kg} / \mathrm{d})$ and prednisolone $(1 \mathrm{mg} / \mathrm{kg} / \mathrm{d})$ has been used for remission-induction therapy of AAV. ${ }^{6}$ Pulsed cyclophosphamide at $15 \mathrm{mg} / \mathrm{kg}$ (maximum of $1.2 \mathrm{~g}$ ) every 2 weeks for the first 3 pulses and every 3 weeks for the following 3-6 pulses in association with prednisolone presented the same induction of remission as continuous oral therapy but with higher risk of relapse. ${ }^{7}$ Recently, rituximab (a monoclonal antibody that binds CD20 protein in $\mathrm{B}$ cells) in association with corticoids has been shown to be as effective as cyclophosphamide as part of induction therapy for AAV with renal involvement, including patients with DAH. ${ }^{8}$ However, this clinical trial excluded patients with severe conditions (creatinine levels of $>3.99 \mathrm{mg} / \mathrm{dL}$ or severe DAH that required mechanical ventilation), so the potential efficacy of rituximab remains unclear in these circumstances. In our case, we used oral cyclophosphamide $(2 \mathrm{mg} / \mathrm{kg} / \mathrm{d})$ with 3 boluses of methylprednisolone $(1 \mathrm{~g} / \mathrm{d}$ followed by $1 \mathrm{mg} / \mathrm{kg} / \mathrm{d})$ with satisfactory results.

There are some reports showing that plasma-exchange therapy improves survival in patients with severe renal vasculitis disease (creatinine levels of $>5.65 \mathrm{mg} / \mathrm{dL}$ ) when used in addition to immunosuppression treatment. ${ }^{9}$ In addition, plasma-exchange therapy may better preserve renal function, with less probability of developing end-stage renal disease. ${ }^{10}$ For DAH, previous studies have demonstrated that a combination of remission-induction therapy and plasma-exchange therapy improves pulmonary infiltrates, respiratory function, and mortality rates, ${ }^{11,12}$ suggesting that active pulmonary hemorrhage could be an indication of early plasmapheresis. However, there is no consensus in the use of plasma-exchange therapy in pulmonary hemorrhage. Currently, the PEXIVAS multi-center randomized controlled trial is evaluating the efficacy of plasmapheresis in combination with corticosteroids in the treatment of AAV. ${ }^{10}$ Our patient had creatinine levels of $<5.65 \mathrm{mg} / \mathrm{dL}$, but active pulmonary hemorrhage was present. Early plasma-exchange therapy with albumin/frozen plasma was started in the 1st day of ICU admission, with improvement of respiratory function.

Respiratory support in severe hypoxemic respiratory insufficiency with $\mathrm{P}_{\mathrm{aO}_{2}} / \mathrm{F}_{\mathrm{IO}_{2}}<150$ with NIV is not well established because of high rates of orotracheal intubation and worse prognosis. ${ }^{13}$ However, Khan et $\mathrm{al}^{3}$ reported the use of NIV in $\sim 40 \%$ of subjects with active GPA who needed respiratory support, with only $14 \%$ requiring orotracheal intubation. Our case suggests that NIV could be effective when aggressive remission-induction therapy is used.

AAV is associated with a higher incidence of venous thromboembolic events. ${ }^{4,5}$ Both deep venous thrombosis and pulmonary emboli are now recognized as important complications of GPA, although the mechanism of this occurrence is unclear. ${ }^{4,14} \mathrm{We}$ tested anti-cardiolipin and 


\section{Pulmonary Thromboembolism in Granulomatosis With Polyangitis}

anti-phospholipid antibodies with negative results. This is in accordance with previous reports demonstrating that the increased risk of venous thromboembolic events is not related to Factor V Leiden nor to anti-cardiolipin and anti$\beta_{2}$-glycoprotein antibodies. ${ }^{14}$ There is no consensus for treatment of venous thromboembolic disease in active GPA. Dreyer et al ${ }^{15}$ described a case of acute GPA treated with standard immunosuppression therapy, which resulted in the development of a massive pulmonary embolism associated with bilateral iliofemoral thrombi. They started subcutaneous enoxaparin and then oral warfarin. The patient later had a pulmonary hemorrhage. Anticoagulation was stopped, a filter was inserted into the vena cava, and 5 plasma exchanges were performed, with improvement of renal and pulmonary function. In contrast, Hughes et al ${ }^{16}$ reported a case of GPA treated successfully with corticosteroids and intravenous cyclophosphamide with excellent clinical response; the subject subsequently developed pulmonary thromboembolic disease. They started anticoagulation that was complicated by severe epistaxis and upper gastrointestinal bleeding with an unsatisfactory outcome.

Active GPA is associated with an increased risk of thromboembolic events and DAH; both presenting simultaneously in a patient is a life-threatening condition. Few data exist for the management of these patients, and there are no recommendations for anticoagulation in acute GPA patients (prophylactic or therapeutic). Vena cava filter insertion is recommended in patients with absolute contraindications for anticoagulation therapy, ${ }^{17}$ so insertion of a vena cava filter may be a better management choice for these patients until disease activity lowers. Aggressive therapy, including plasma exchange, is necessary to treat these patients because lowering disease activity improves DAH but also diminishes the incidence of thromboembolic events. All of these issues should be studied in clinical trials to improve the ICU management of these patients in the context of active disease.

\section{Teaching Points}

- Active GPA vasculitis increases the incidence of thromboembolic events. Pulmonary embolism in association with DAH in GPA is a life-threatening condition.

- Aggressive remission-induction therapy is indicated for patients with GPA and DAH, including boluses of corticosteroids at high doses and then $1 \mathrm{mg} / \mathrm{kg} / \mathrm{d}$ and oral cyclophosphamide at $2 \mathrm{mg} / \mathrm{kg} / \mathrm{d}$.

- Although not well established, we suggest the use of plasma-exchange therapy to treat these patients because of the severity of condition.

- Anticoagulation cessation, intra-arterial subtraction angiography, and vena cava filter insertion until the activity of the disease diminishes is probably the most appropriate treat- ment strategy for patients with active GPA and DAH with deep venous thrombosis and pulmonary embolism.

- Despite few reports of the use of NIV for DAH in active GPA, it could be used until the activity of the disease decreases and respiratory function improves.

\section{REFERENCES}

1. Hauer HA, Bajema IM, van Houwelingen HC, Ferrario F, Noël LH, Waldherr R, et al. Renal histology in ANCA-associated vasculitis: differences between diagnostic and serologic subgroups. Kidney Int 2002;61(1):80-89.

2. West S, Arulkumaran N, Ind PW, Pusey CD. Diffuse alveolar haemorrhage in ANCA-associated vasculitis. Intern Med 2013;52(1):5-13.

3. Khan SA, Subla MR, Behl D, Specks U, Afessa B. Outcome of patients with small-vessel vasculitis admitted to a medical ICU. Chest 2007;131(4):972-976.

4. Allenbach Y, Seror R, Pagnoux C, Teixeira L, Guilpain P, Guillevin L. High frequency of venous thromboembolic events in Churg-Strauss syndrome, Wegener's granulomatosis and microscopic polyangiitis but not polyarteritis nodosa: a systematic retrospective study on 1130 patients. Ann Rheum Dis 2009;68(4):564-567.

5. Merkel PA, Lo GH, Holbrook JT, Tibbs AK, Allen NB, Davis JC Jr, et al. Brief communication: high incidence of venous thrombotic events among patients with Wegener granulomatosis: the Wegener's Clinical Occurrence of Thrombosis (WeCLOT) Study. Ann Intern Med 2005;142(8):620-626.

6. Mukhtyar C, Guillevin L, Cid MC, Dasgupta B, de Groot K, Gross $\mathrm{W}$, et al. EULAR recommendations for the management of primary small and medium vessel vasculitis. Ann Rheum Dis 2009;68(3): 310-317.

7. Harper L, Morgan MD, Walsh M, Hoglund P, Westman K, Flossmann O, et al. Pulse versus daily oral cyclophosphamide for induction of remission in ANCA-associated vasculitis: long-term followup. Ann Rheum Dis 2012;71(6):955-960.

8. Stone JH, Merkel PA, Spiera R, Seo P, Langford CA, Hoffman GS, et al. Rituximab versus cyclophosphamide for ANCA-associated vasculitis. N Engl J Med 2010;363(3):221-232.

9. Jayne DR, Gaskin G, Rasmussen N, Abramowicz D, Ferrario F, Guillevin L, et al. Randomized trial of plasma exchange or highdosage methylprednisolone as adjunctive therapy for severe renal vasculitis. J Am Soc Nephrol 2007;18(7):2180-2188.

10. Casian A, Jayne D. Plasma exchange in the treatment of Wegener's granulomatosis, microscopic polyangiitis, Churg-Strauss syndrome and renal limited vasculitis. Curr Opin Rheumatol 2011;23(1):12-17.

11. Aydin Z, Gursu M, Karadag S, Uzun S, Tatli E, Sumnu A, et al. Role of plasmapheresis performed in hemodialysis units for the treatment of anti-neutrophilic cytoplasmic antibody-associated systemic vasculitides. Ther Apher Dial 2011;15(5):493-498.

12. Sugimoto T, Deji N, Kume S, Osawa N, Sakaguchi M, Isshiki K, et al. Pulmonary-renal syndrome, diffuse pulmonary hemorrhage and glomerulonephritis, associated with Wegener's granulomatosis effectively treated with early plasma exchange therapy. Intern Med 2007;46(1):49-53.

13. Thille AW, Contou D, Fragnoli C, Córdoba-Izquierdo A, Boissier F, Brun-Buisson C. Non-invasive ventilation for acute hypoxemic respiratory failure: intubation rate and risk factors. Crit Care 2013; 17(6):R269.

14. Sebastian JK, Voetsch B, Stone JH, Romay-Penabad Z, Lo GH, Allen NB, et al. The frequency of anticardiolipin antibodies and genetic mutations associated with hypercoagulability among patients with Wegener's granulomatosis with and without history of a thrombotic event. J Rheumatol 2007;34(12):2446-2450. 


\section{Pulmonary Thromboembolism in Granulomatosis With Polyangitis}

15. Dreyer G, Fan S. Therapeutic implications of coexisting severe pulmonary hemorrhage and pulmonary emboli in a case of Wegener granulomatosis. Am J Kidney Dis 2009;53(5):e5-e8.

16. Hughes M, Burgess J, Parekh N. Wegener's granulomatosis complicated by pulmonary embolism: a case report and review of the literature. Mod Rheumatol 2011;21(2):211-214.
17. Jaff MR, McMurtry MS, Archer SL, Cushman M, Goldenberg N, Goldhaber SZ, et al. Management of massive and submassive pulmonary embolism, iliofemoral deep vein thrombosis, and chronic thromboembolic pulmonary hypertension: a scientific statement from the American Heart Association. Circulation 2011;123(16):17881830 . 